\title{
Paratenon of the cruciate ligaments of the knee: a macroscopic and histological study of human foetuses
}

\author{
J.H. Kim¹, N. Sugai², D. Suzuki³ , G. Murakami ${ }^{4}$, H. Abe ${ }^{5}$, J.F. Rodríguez-Vázquez ${ }^{6}$, \\ M. Yamamoto ${ }^{7}$

\begin{abstract}
${ }^{1}$ Department of Anatomy, Jeonbuk National University Medical School, Jeonju, Korea 2Department of Rehabilitation and Physical Therapy, Hitshuji-ga-oka Hospital, Sapporo, Japan ${ }^{3}$ Division of Common Curriculum, Hokkaido Chitose College of Rehabilitation, Chitose, Japan ${ }^{4}$ Division of Internal Medicine, Cupid Clinic, Iwamizawa, Hokkaido, Japan ${ }^{5}$ Emeritus Professor of Akita University School of Medicine, Akita, Japan ${ }^{6}$ Department of Anatomy and Embryology, School of Medicine, Complutense University, Madrid, Spain 'Department of Anatomy, Tokyo Dental College, Tokyo, Japan
\end{abstract}

[Received: 8 December 2020; Accepted: 4 January 2021; Early publication date: 22 January 2021]

Background: The paratenon is a sheath-like connective tissue that allows the tendon to move with minimal friction. The careful removal of the paratenon along the cruciate ligaments is a critical step of knee surgery. Thus, orthopaedic surgeons and interventional radiologists consider the paratenon as a basic anatomical tissue along a ligament, not along a tendon.

Materials and methods: We performed macroscopic and histological observations of cruciate ligament-associated paratenons in 43 human foetuses.

Results: This tissue usually had a thick armour-like appearance that was distant from the infrapatellar fat pad. The anterior cruciate ligament, rather than the posterior ligament, was deeply embedded in the paratenon. The paratenon contained abundant arteries and veins and, at and near the crossing between the cruciate ligaments, had a well-developed venous plexus. Notably, there were abundant fused veins in the paratenon venous plexus, and prenatal knee movements (especially rotation) seemed to restrict its blood supply, leading to the development of a large cavity by way of advancing fusion of veins in the degenerating plexus. This unique manner of cavitation likely expanded the joint cavity.

Conclusions: Differences in knee movements in utero seemed to cause differences in the thickness of the paratenon among foetuses. New-borns might have limited knee flexion due to a mass-effect of the thick paratenon around the cruciate ligaments. A slight twisting or rotation at the knee may help to release the knee, because it can break the foetal paratenon and accelerate cavitation. (Folia Morphol 2022; 81, 1: 134-143)

Key words: paratenon, tendon sheath, cruciate ligament of the knee, histology, human foetus 


\section{INTRODUCTION}

There is variation and some confusion in use of the term "paratenon" among clinicians. Benjamin et al. [1] provided concise definitions of the paratenon and its analogues as follows: the endotendon is a thin film of loose connective tissue providing a vascular route; the epitenon surrounds the tendon as a whole and is continuous with the endotendon; and the paratenon is a tendon sheath that has a different histology and is clearly separated from the tendon itself. These authors considered the paratenon of the Achilles tendon as the best example. Therefore, the paratenon is not usually continuous with the synovial lining of a joint; similarly, some bursas, such as the trochanteric bursa, do not communicate with the joint cavity.

The careful removal of the paratenon along the cruciate ligament is a critical step in knee surgery [3], and paratenon stripping is an important procedure in interventional radiology of the knee [14]. The paratenon of the knee refers specifically to a sheath of the ligament, in contrast to the other or more common use of this term to refer to a sheath of the Achilles tendon $[8,18,19]$. Clinicians commonly use the term "paratenonitis" to refer to inflammation of the outer layer of the tendon, regardless of whether the paratenon is lined by a synovium [12]. However, for knee surgeons and interventional radiologists, the paratenon is a basic anatomical tissue along a ligament, not along a tendon. In the knee joint, the synovial lining should cover the paratenon along the cruciate ligaments. Because the infrapatellar fat pad is the largest synovial plica (thin tissue fold) in the human body, it might communicate with the paratenon.

The general aim of this study was to describe the development and morphology of the paratenon along the cruciate ligaments of the knee in human foetuses. The cruciate ligaments develop in the interzone mesenchymal tissue, between the femur and tibia $[5,15,16]$. Thus, it is likely that the paratenon also develops from primary interzone tissue, rather than being divided from the established cruciate ligament. The specific aim of this study was to determine whether a synovial lining was present at the expanding joint cavity along and around the paratenon of the knee. Previous research showed that the cytoplasm-rich cells in foetal synovial tissues had a cuboidal appearance $[9,13]$.

\section{MATERIALS AND METHODS}

This study was performed in accordance with the provisions of the 2013 revision of the Declaration of Helsinki. For macroscopic observations, the left knees of 25 near-term foetuses (crow-rump length [CRL]: 272-310 mm; gestational age [GA]: 32-37 weeks) were examined. The patellar tendon was cut at the tibial attachment to reflect the patella and infrapatellar fat pad upward. During the reflection, an attachment of the fat pad to the femur was cut (if present). A slight dissection was performed along the cruciate ligaments, and photos were taken at each step using a Pentax K-1 camera with a $50-100 \mathrm{~mm}$ zoom lens.

For histological observations, sagittal sections of the unilateral knee from 8 midterm foetuses (CRL: 35-73 mm; GA: 8-12 weeks) and 10 near-term foetuses (CRL: 280-320 mm; GA: 33-39 weeks) were examined. The latter 10 specimens were not used for the macroscopic observations. All sections were stained with haematoxylin and eosin (H\&E). Most histological photographs were taken using a Nikon Eclipse 80, but photographs at ultra-low magnification (objective lens less than $\times 1$ ) were taken using a high-grade flat scanner with translucent illumination (Epson scanner GTX970).

The histological sections from 6 midterm foetuses were part of the large collection at the Department of Anatomy of the Universidad Complutense, Madrid, and were from miscarriages or ectopic pregnancies at the Department of Obstetrics of the University. Until 1980, the sections had been prepared and stained with H\&E, Azan or silver impregnation. The used of these specimens for research was approved by the Ethics Committee of Complutense University (B08/374). The knees of near-term foetuses were part of the collection of the Department of Anatomy, Akita University, Japan and were donated by families to the Department in 1975-1985 and preserved in $10 \% \mathrm{w} / \mathrm{w}$ neutral formalin solution for more than 30 years. Data on these specimens included the date of donation and the number of gestational weeks, but not the name of the family, obstetrician, hospital, or reason for abortion. The use of these specimens for research was approved by the Akita University Ethics Committee (No. 1428).

The unilateral knees from the 10 near-term foetuses were divided, and the specimens were incubated at room temperature in Plank-Rychlo solution $\left(\mathrm{AlCl}_{2} / 6 \mathrm{H}_{2} \mathrm{O}, 7.0 \mathrm{w} / \mathrm{v} \% ; \mathrm{HCl}, 3.6 \% ; \mathrm{HCOOH}, 4.6 \%\right.$ ) for 1 to 2 weeks. Routine procedures for paraffin-embedded histology were performed, and semiserial sections of knees were prepared at $0.1 \mathrm{~mm}$ intervals. 

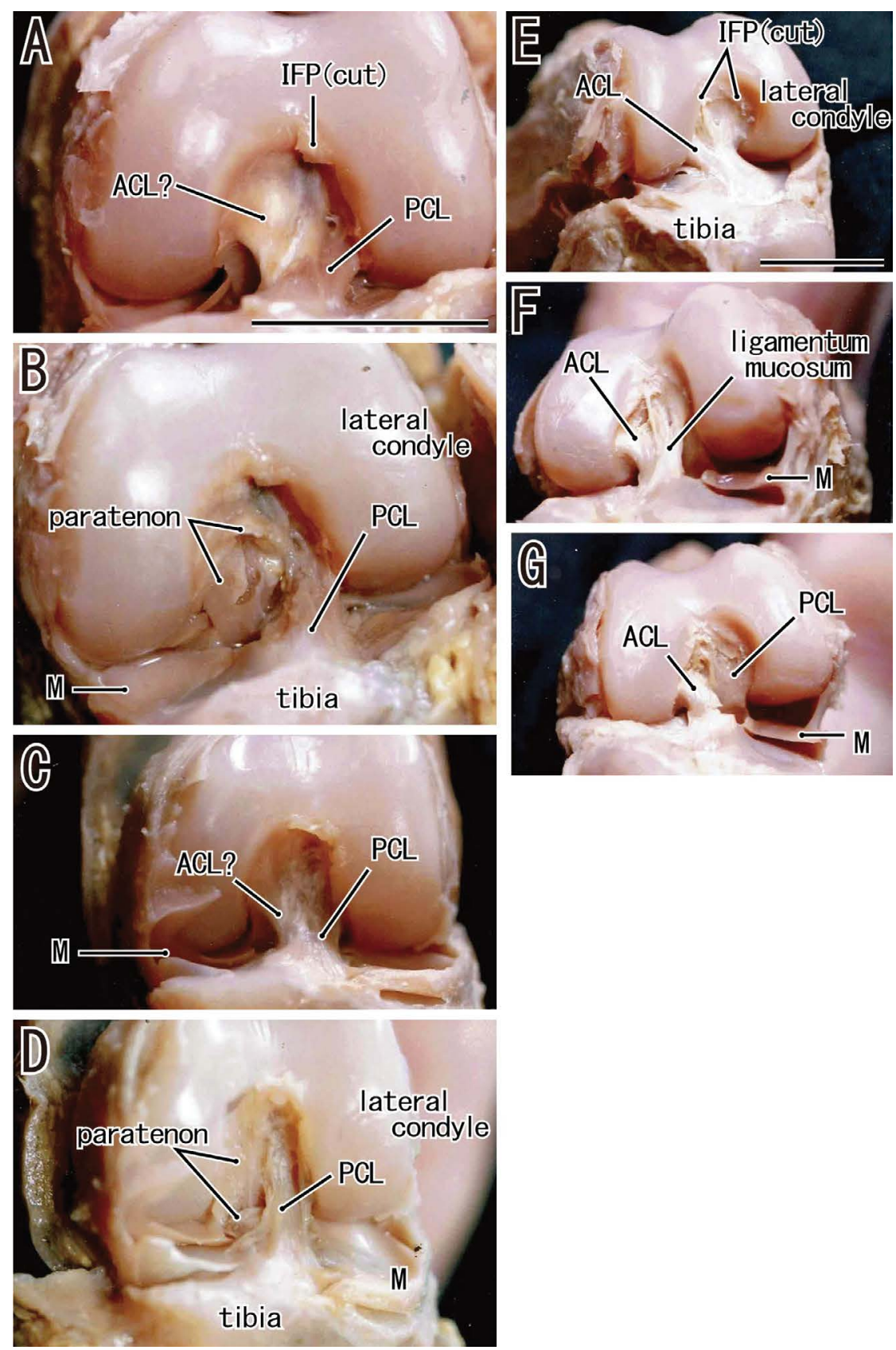

Figure 1. Anterior macroscopic views of the left knees of three near-term foetuses, with the patellas, infrapatellar fat pads, and patellar tendons removed; A, B. Foetus of $305 \mathrm{~mm}$ crow-rump length (CRL), approximately 36 weeks gestational age (GA); C, D. Foetus of $286 \mathrm{~mm} \mathrm{CRL,}$ approximately 33 weeks GA; E-G. Foetus of $276 \mathrm{~mm}$ CRL, approximately 32 weeks GA. When the knee joint was opened, the infrapatellar fat pad was cut at the femoral attachment if present (infrapatellar fat pad [IFP] cut, panel A). The anterior cruciate ligaments (ACL) was entirely covered by a thick capsule-like paratenon (panel B), and the paratenon likely contained a feather-like structure (panel D). Panel E shows a rare example of a thin paratenon along the anterior ligament. The posterior cruciate ligament (PCL) accompanied a band-like tissue (ligamentum mucosum, panel F). Both ligaments were almost in parallel when the tibia was twisted (panel G); M - meniscus. Scale bar in panel A ( $5 \mathrm{~mm}$ ) refers to panels $A-D$; scale bar in panel $E(5 \mathrm{~mm})$ refers to panels $E-G$.

\section{RESULTS}

\section{Macroscopic observations}

When we removed the patella and patellar tendon to open the knee joint from the anterior side, we also pulled off the infrapatellar fat pad from the joint cavity because it was attached to the patellar tendon at the inferior side of the patella. However, because a marginal part of the fat pad was attached to the femur, we cut it before taking photos (Fig. 1). The cruciate ligaments consistently had a smooth 
surface (Fig. 1A, C, E). However, this surface was not usually of the ligament itself, but of the paratenon. Twenty-three of the 25 knees examined macroscopically had anterior cruciate ligaments (ACLs) that were entirely covered by capsule-like paratenons (Fig. 1B), and these sometimes contained feather-like structures attached to the anterior aspect of the ACL (Fig. 1D). Thus, in contrast to the first impression upon opening of the knee, the actual $A C L$ was rarely exposed to the joint cavity (Fig. 1E). The ACL was evident in the anterior view in the other two knees (Fig. 1E, F). When the tibia was twisted, a crossing between the $\mathrm{ACL}$ and the posterior cruciate ligament $(\mathrm{PCL})$ was released, and provided an almost parallel arrangement (Fig. 1G). This posture allowed us to determine whether a residual paratenon was present or absent after dissection.

The paratenon also covered the $\mathrm{PCL}$, but was usually much thinner there than along the ACL. In 6 of 25 knees in which the paratenon was thick along the PCL, it had the appearance of another ligament that merged with the $A C L$ at the tibial attachment (i.e. the ligamentum mucosum, Fig. 1F). Therefore, in these 6 knees, the paratenon of the PCL tended to be thicker on the lower side due to the antero-inferior course of the ligamentum mucosum. We found no correlation between the foetal age and gross morphology of the paratenon.

\section{Histological observations}

At midterm, an interzone mesenchymal tissue between the femur and tibia gradually disappeared (Fig. 2A). However, at 9 weeks GA, most parts of the $A C L$ were embedded in the interzone tissue remnant (Fig. 2B). At that time, a joint cavitation advanced into the posterolateral part. Thus, along the $\mathrm{PCL}$, the interzone tissue was limited to near the femoral attachment (Fig. 2C). Development of the infrapatellar fat pad was much delayed relative to the cruciate ligaments (Fig. 2B).

At near-term, we found various stages of paratenon development, and no clear correlation of stage with foetus age or size. Thus, the larger knees did not usually have a sheath- or armour-like paratenon, and also did not usually have a larger joint cavity. A candidate initial stage of the paratenon differentiation was present in the posterior side of the ACL (Fig. 3A, B). In this case, the enlarging joint cavity was surrounded by synovial plica-like tissues and by ligament-like tight tissues; the latter was apparently divided from the

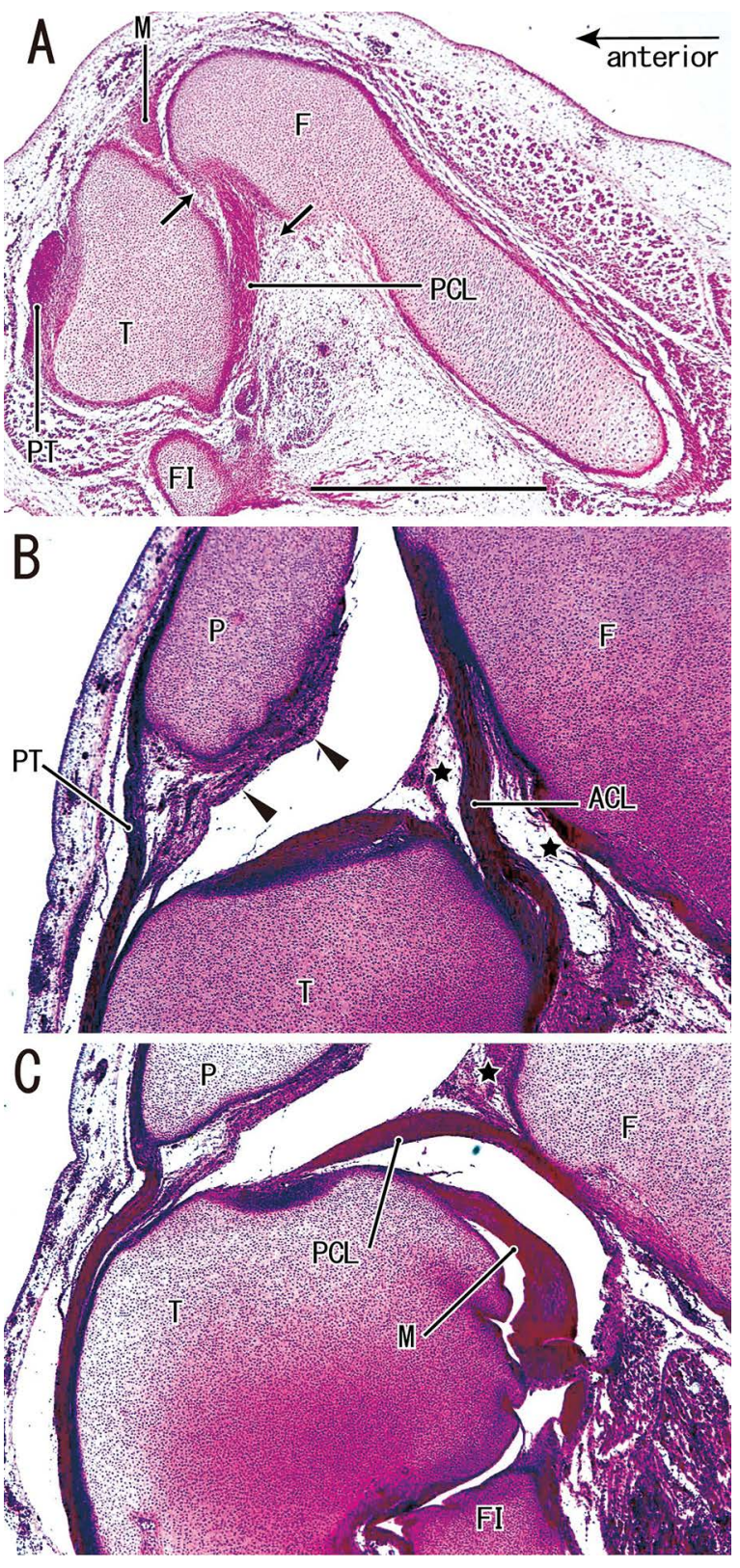

Figure 2. Sagittal sections of interzone mesenchymal tissues of the knee joints of two midterm foetuses; A. Foetus of $35 \mathrm{~mm}$ crow-rump length (CRL), approximately 8 weeks gestational age (GA); B, C. Foetus of $46 \mathrm{~mm} \mathrm{CRL,} \mathrm{approximately} 9$ weeks GA. At 8 weeks, the posterior cruciate ligament $(\mathrm{PCL})$ was embedded in interzone mesenchymal tissue (arrows in panel A) of the knee joint. The patella was present in more medial sections. At 9 weeks, the anterior cruciate ligament $(A C L)$ was embedded in the interzone tissue remnant (stars in panel B) along almost the entire course. In contrast, along the PCL the loose tissue (star in panel $\mathrm{C}$ ) was restricted near the femur (F). The infrapatellar fat pad was not developed (arrowheads in panel $\mathrm{B}$ ); $\mathrm{FI}$ - fibula; $\mathrm{M}$ - meniscus; $\mathrm{P}$ - patella; $\mathrm{PT}$ patellar tendon; $T$ - tibia. Scale bar in panel $A(1 \mathrm{~mm})$ refers to all panels.

ACL with long tears (Fig. 3B). In contrast, an initial change was limited to an interzone tissue remnant that extended inferiorly along the PCL (Fig. 3C, D). 

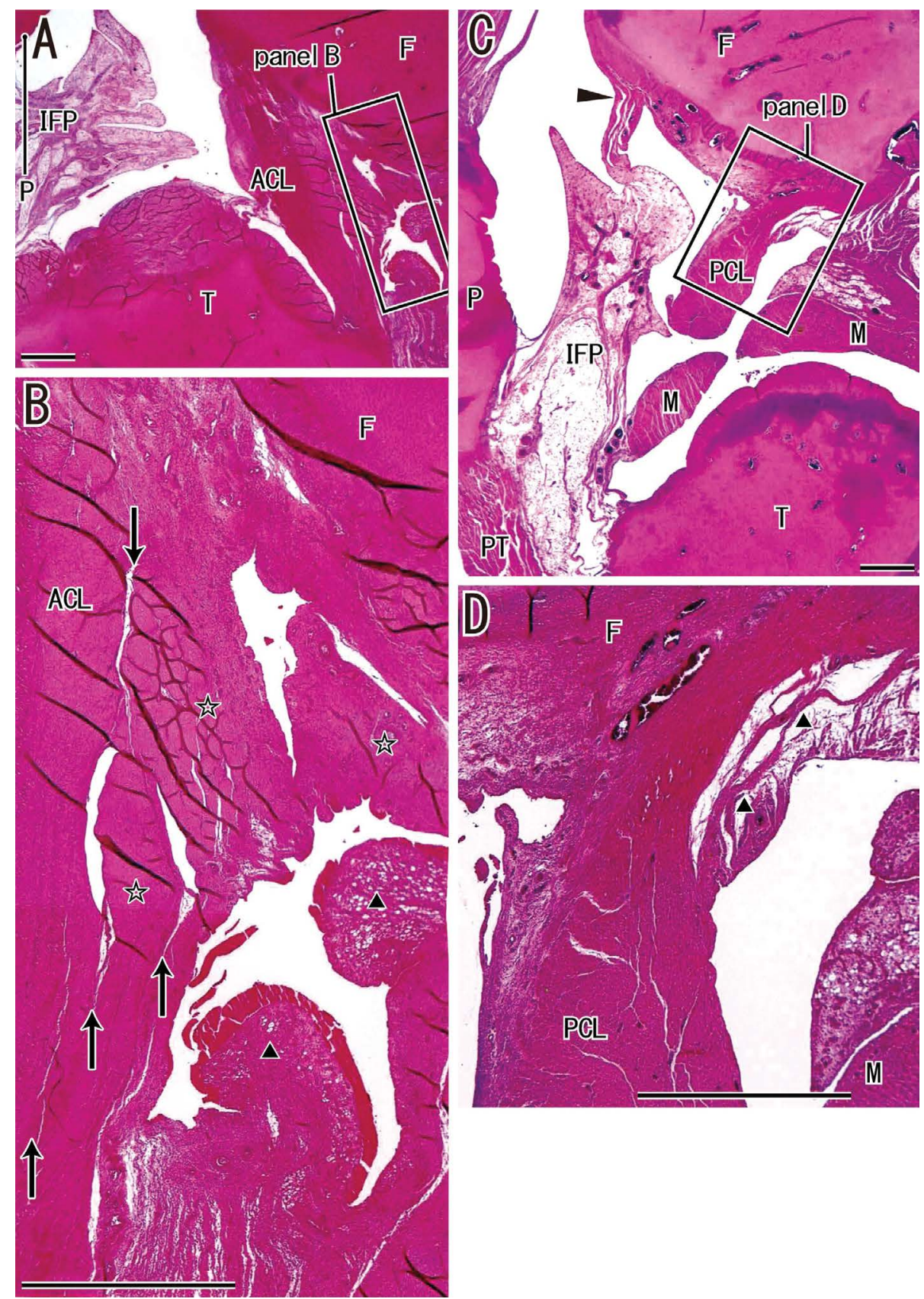

Figure 3. Sagittal sections of the initial differentiation of the paratenon along the cruciate ligaments of the knees of two near-term foetuses; A, B. Foetus of $270 \mathrm{~mm}$ crow-rump length (CRL), approximately 32 weeks gestational age (GA); C, D. Foetus of $282 \mathrm{~mm}$ CRL, approximately 33 weeks $G A$. Panels $B$ and D provide magnified views of the delineated regions in panels $A$ and $C$, respectively. An expanding joint space in the posterior side of the anterior cruciate ligaments (ACL) was surrounded by synovial fold-like tissues (triangles in panel $B$ ) and by ligament-like tight tissues (stars in panel B). The latter appear to be divided from the anterior ligament because of tears (arrows in panel B). In contrast, a loose tissue (triangles in panel D), corresponding to an interzone tissue remnant (Fig. 2C), extended inferiorly along the posterior cruciate ligament (PCL). The infrapatellar fat pad (IFP) was connected to the femur (arrowhead in panel C); $\mathrm{F}$ - femur; $\mathrm{M}$ - meniscus; $\mathrm{P}$ - patella; PT — patellar tendon; T — tibia. Scale bar in panel A $(1 \mathrm{~mm})$ refers to panels $A$ and $C$; scale bar in panel $B(1 \mathrm{~mm})$ refers to panels $B$ and $D$.

At the next stage (CRL $320 \mathrm{~mm}$ ), the $\mathrm{ACL}$ accompanied a fatty tissue-rich paratenon or synovial plica around it (Fig. 4) or was embedded in a venous plexus-like paratenon (Fig. 6). These loose tissues contained abundant veins and several arteries. In contrast, the PCL accompanied the paratenon at and near the bony attachments. The infrapatellar fat pad was thick and long, and composed of a mesh of fibrous bands (less than $0.1 \mathrm{~mm}$ thick) that contained fatty tissues between the bands (Fig. 4C). The fat pad 

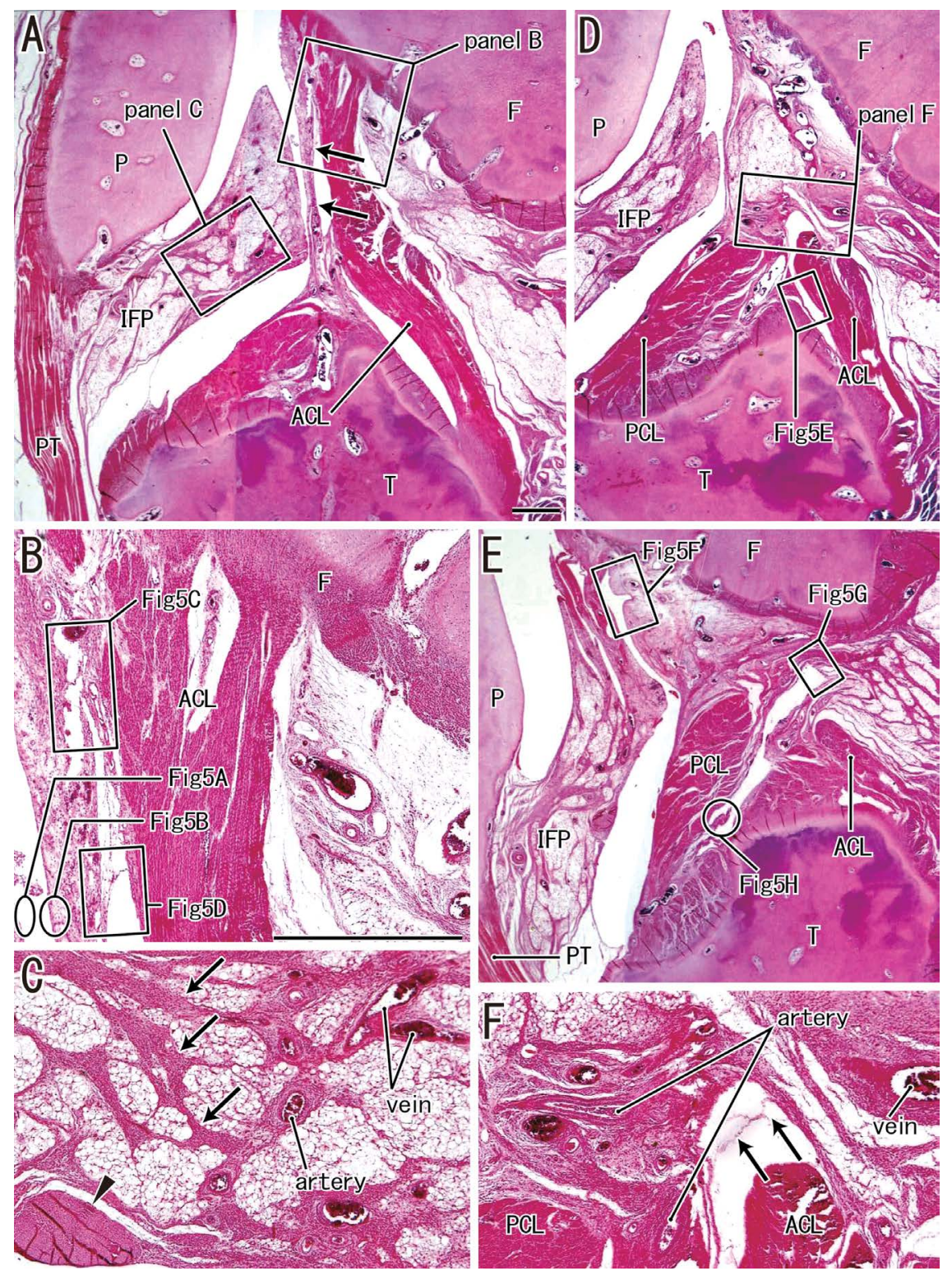

Figure 4. Sagittal sections of the fibro-adipose tissues of the paratenon at the knee in a near-term foetus; A-F. Foetus of $320 \mathrm{~mm}$ crow-rump length (CRL), approximately 39 weeks gestational age (GA). Panels $A$ (most medial), $D$, and $E$ (most lateral) show the anterior cruciate ligament (ACL) and posterior cruciate ligament (PCL) at low magnification. The ACL is separated from the infrapatellar fat pad (IFP) by a synovial plica (arrows in panel A). Panels B, C, and F provide magnified views of the delineated regions in panels $A$ and $B$, and Figure 5 provides magnified views of the delineated regions in panels $B$ and $E$. The femoral attachment of the anterior ligament (panel $B$ ) was sandwiched by loose tissues or paratenons. Panel $\mathrm{C}$ shows the infrapatellar fat pad, which consists of fatty tissues in a mesh of fibrous bands (arrows; $<0.1 \mathrm{~mm}$ thick). Some fat pads contained thick fibre bundles (arrowhead; $>0.3 \mathrm{~mm}$ thick). Panel F shows a site near the crossing of the cruciate ligaments, in which the arteries run near the ligaments. A cavity along the anterior ligament contained remnant fibres (arrows in panel F). $\mathrm{F}$ - femur; $\mathrm{P}$ - patella; $\mathrm{PT}$ - patellar tendon; $\mathrm{T}$ - tibia. Scale bar in panel $\mathrm{A}(1 \mathrm{~mm})$ refers to panels $\mathrm{A}, \mathrm{D}$ and $\mathrm{E}$; scale bar in panel $\mathrm{B}(1 \mathrm{~mm})$ refers to panels $B, C$ and $F$.

surface and the paratenon surface to the joint cavity were lined by cuboidal cells (i.e. a primitive synovial lining, Fig. 5A, B, F). In contrast, flat cells lined a loose space along the $A C L$ (Fig. 5C, D).

Notably, the cavity with flat lining cells continued to or communicated with the other non-lining spaces, which contained thin fibres (Figs. $4 \mathrm{~F}$ and $5 \mathrm{E}$ ) and/or fragmented tissues (Fig. 6C, D, G). Thus, at near-term, the drastic reconstruction of the paratenon was likely to occur with a cavitation along the ACL. Likewise, in the venous plexus-like paratenon (Fig. 7), vein fusion and degeneration provided a venous valve-like struc- 


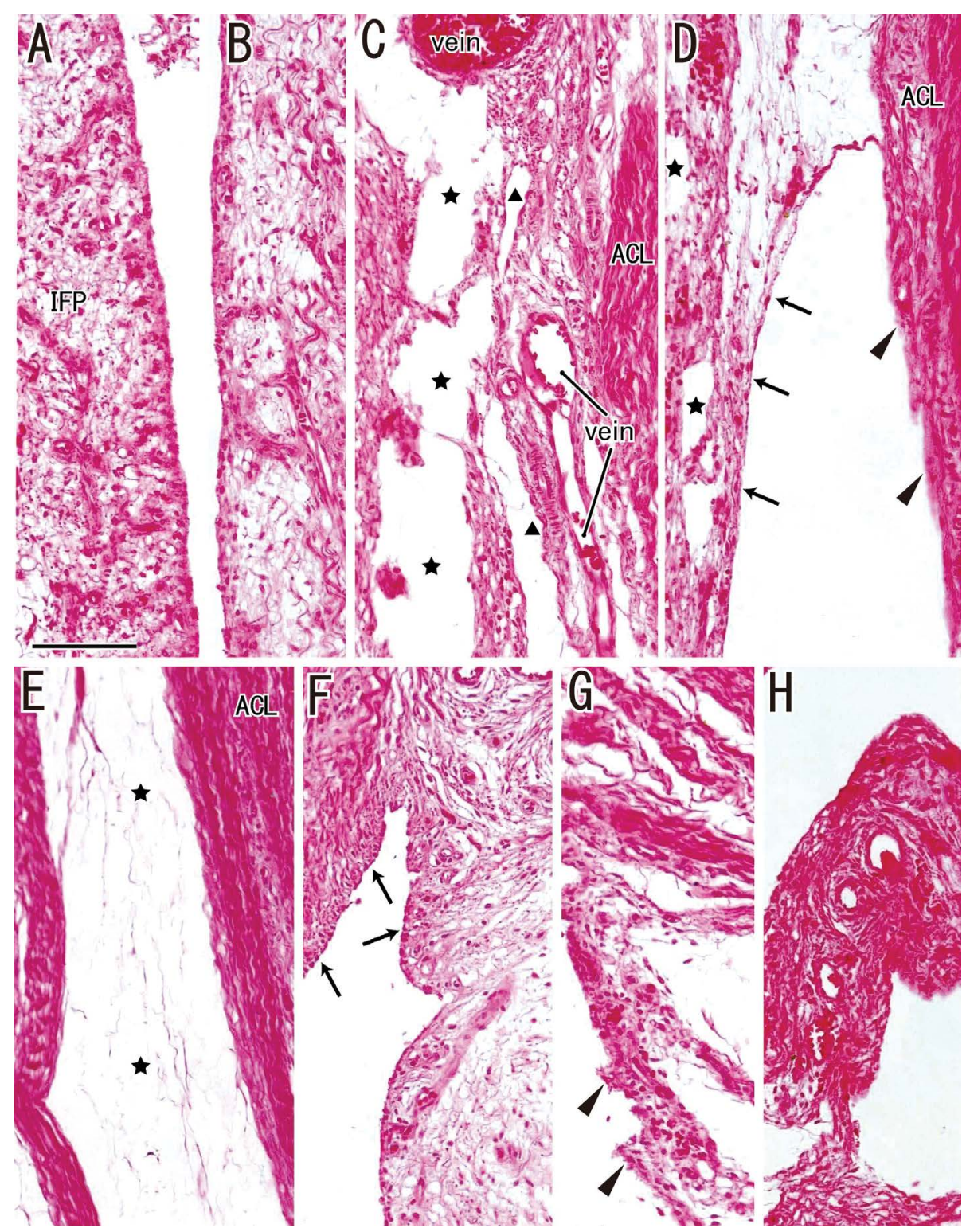

Figure 5. A-H. Higher magnification views of the fibro-adipose paratenon and synovium in a near-term foetus. Images are from delineated regions in panels $B, D$ and $E$ of Figure 4. Cuboidal and flat cells were mixed, and lined a surface of the infrapatellar fat pad (IFP, panel A) and a synovial plica along the anterior ligament (panel B). Along the anterior aspect of the anterior cruciate ligament (ACL) (panel C), there was a thick and long cavity without lining cells (stars) and two thin cavities with a lining of flat cells (triangles). At a site below panel C, a cavity in panel $\mathrm{D}$ was lined by flat cells (arrows) but was exposed to ACL fibres without lining (arrowheads). There were also small spaces with unclear lining cells (stars in panel D). Near the tibial attachment of the ACL (panel E), a cavity (stars) contained multiple remnant fibres. There was a cuboidal cell lining of the paratenon near the femoral attachment of the anterior ligament (arrows in panel F). In contrast, there were fragmented tissues without lining cells (arrowheads in panel G) near the femoral attachment of the posterior cruciate ligament (PCL). The PCL had a short synovial plica near the tibia (panel H). Scale bar in panel A $(1 \mathrm{~mm})$ refers to all panels.

ture (i.e. a degenerating venous endothelium, Fig. $7 B-E)$. This morphology was quite different from an actual vein that was surrounded by a flat endothelial lining (Fig. 7A). Therefore, the venous fusion was similar to the process of joint cavitation via fusion of small spaces in degenerative tissues (Fig. 5C). These processes of the cavitation and venous reconstruction in the paratenon exposed the nearby arteries to the joint cavity (Figs. $4 \mathrm{~F}$ and $6 \mathrm{G}$ ).

\section{DISCUSSION}

The present study is apparently the first histological report of the paratenon of the knee in human foetuses. Because these ligament-associated 

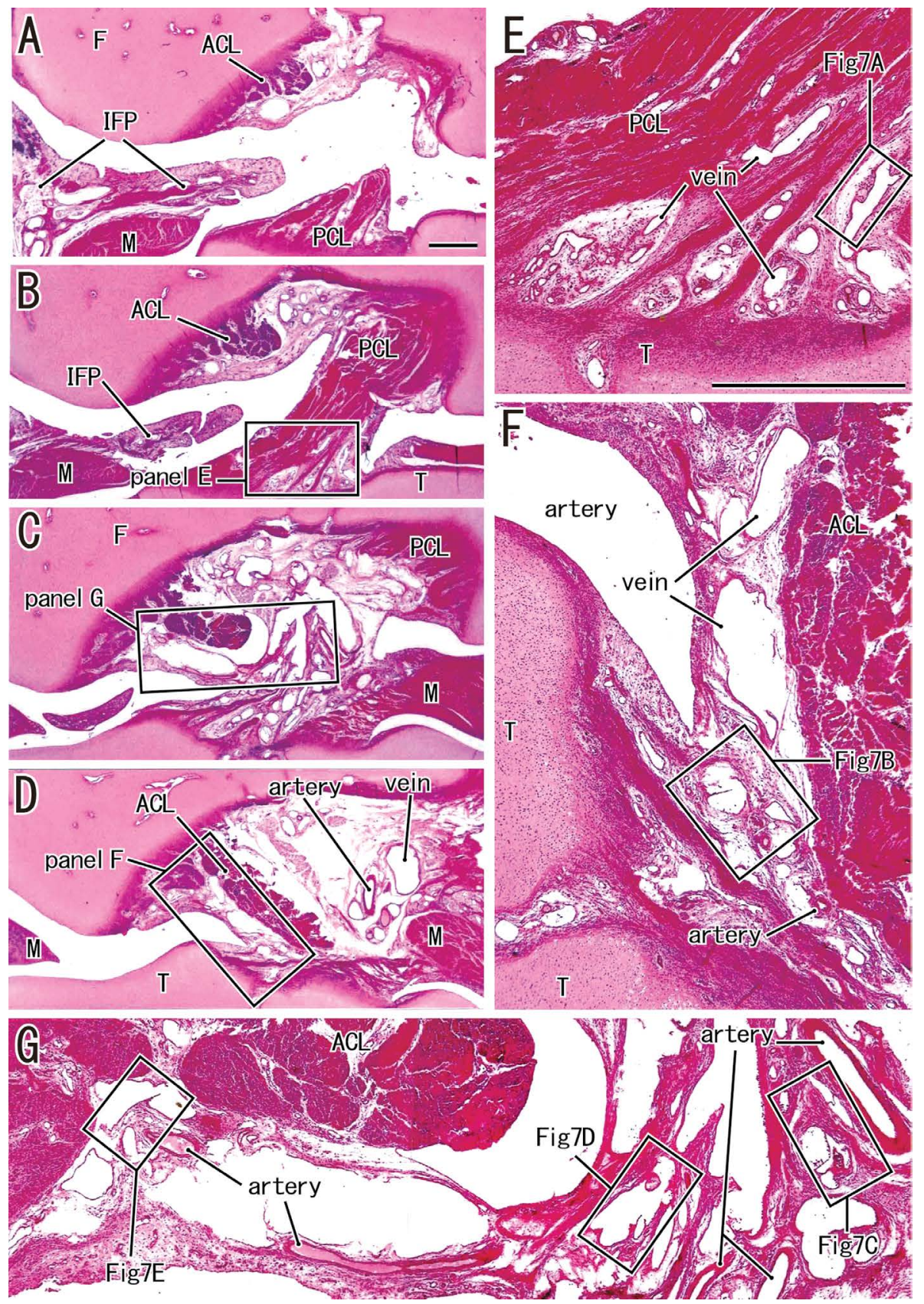

Figure 6. Sagittal sections of a vein-rich paratenon at the knee in a near-term foetus $(280 \mathrm{~mm}$ crow-rump length, approximately 33 weeks gestational age); A-D. The anterior cruciate ligament ( $A C L$ ) and posterior cruciate ligament (PCL) at low magnification, and panel $A$ is the most medial view. Delineated areas in these panels are shown in panels E-G and Figure 7. The tibial attachment of the posterior ligament (panel E) contains abundant veins. In the anterior side of the anterior ligament (panel F), veins in the paratenon appear to be broken or degenerated (see also Fig. 7B). Near the crossing of the ACL and PCL (panel G), veins with irregular shapes form a plexus (see also Fig. 7C-E). $\mathrm{F}$ - femur; IFP — infrapatellar fat pad; $\mathrm{M}$ - meniscus; $\mathrm{T}$ - tibia. Scale bar in panel A $(1 \mathrm{~mm})$ refers to panels A-D; scale bar in panel $\mathrm{E}$ $(1 \mathrm{~mm})$ refers to panels $\mathrm{E}-\mathrm{G}$.

tissues are small and thin in midterm foetuses, we believe that the thick paratenons that we identified macroscopically in near-term foetuses (Fig. 1) likely developed from the interzone mesenchymal tissue between 13 and 31 weeks GA. In contrast to the development of a tight meniscus in the loose interzone tissue [4], a relatively loose paratenon did not have a definite shape during the prenatal period. However, in near-term foetuses, there was likely to be a drastic reconstruction of the paratenon, especially along the ACL. At this time, individual differences in the thickness of the paratenon became evident along the 


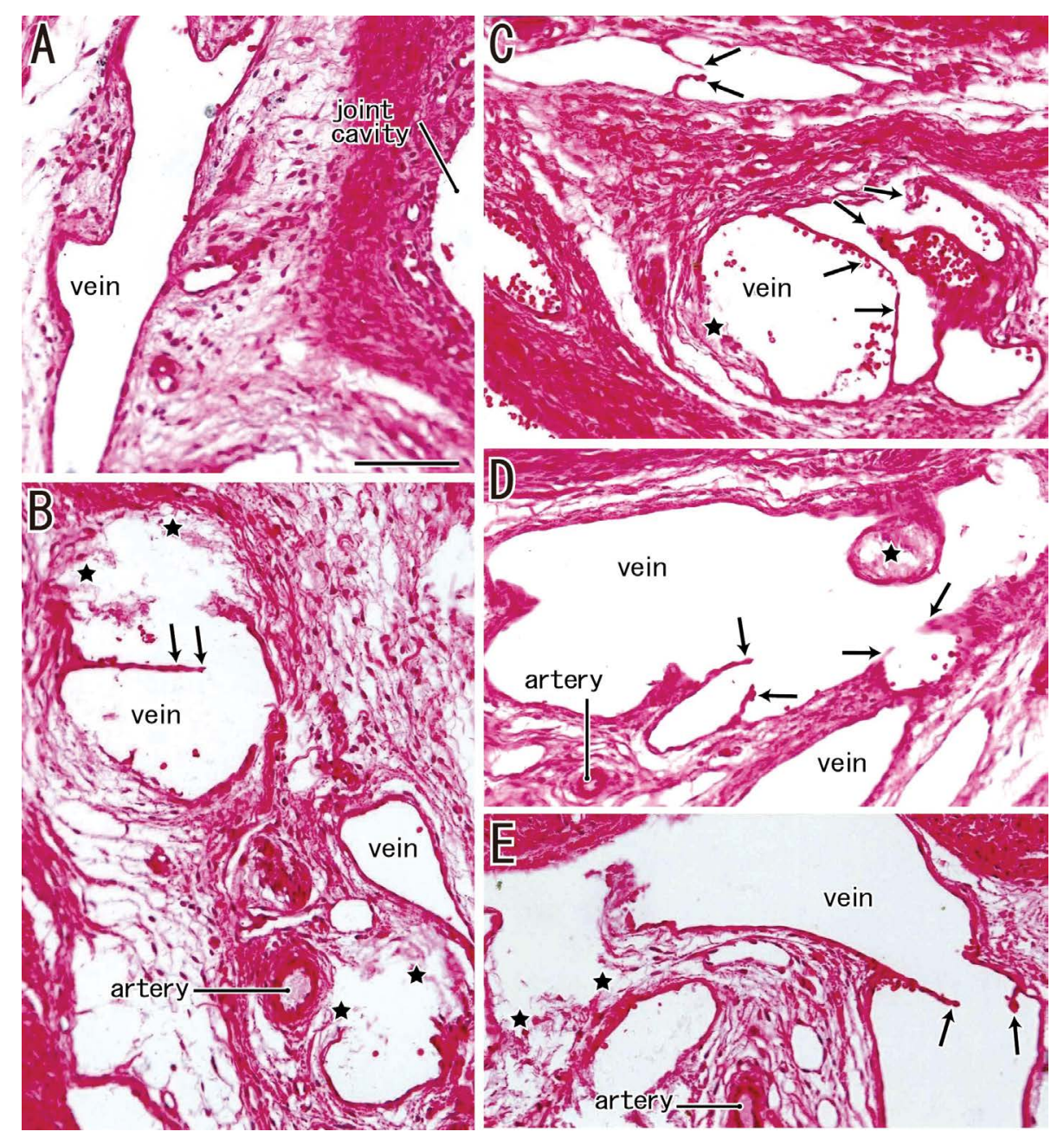

Figure 7. A-E. Higher magnification views of normal and specific veins in the paratenon of a near-term foetus. Images are from delineated regions in panels E-G of Figure 6. Panel A shows a flat endothelial cell lining of a vein that is clearly different from the synovial lining of the joint cavity (see also Fig. 5A, B). Veins in the paratenon of the cruciate ligaments (panels B-E) often have valve-like structures of the endothelium (arrows) and broken or degenerated endothelium (stars). Scale bar in panel $A(0.1 \mathrm{~mm})$ refers to all panels.

$\mathrm{ACL}$, presumably due to differences in knee posture in utero. A slight twisting of the cruciate ligaments (i.e. a slight rotation at the knee) at this time probably pushed and interfered with enlargement of the paratenon. Actually, Katz et al. [10] reported greater external rotation of the tibia in babies after earlier delivery. We found two-types of histology: a fatty tissue-rich paratenon and a venous plexus-like paratenon. Because active cavitation in the paratenon was suggested in the venous plexus, it is unlikely that one type of paratenon could change into the other type. In both types, the paratenon contained abundant arteries and veins, especially at and near the crossing between the cruciate ligaments.

Notably, veins in the paratenon were likely to fuse and, because of limited blood supply from prenatal knee movements, the plexus seemed to degenerate.
In the degeneration in near-term knees, the advancing fusion of venous cavities seemed to provide a large cavity without the usual cuboidal synovial cell lining. This might be a unique manner of joint cavitation, although it is somewhat similar to the fusion of thyroid follicles in the aged thyroid because of abundant valve-like fragments of the follicle wall [20]. A similar fusion is also seen in circadian changes of the follicles although it is followed by re-division: the size of follicle drastically changes [21]. The unique cavitation in the paratenon venous plexus exposed the nearby arteries to the joint cavity (Figs. 4F and 6G), and these arteries should be degenerated. In near-term foetuses, we recently reported a close topographical relationship between the tributaries of the external vertebral venous plexus and recesses of the zygapophysial joint cavity [13]. A degenerating venous 
plexus during joint cavitation might be a common method of development. Although we cannot deny the possibility that the present venous plexus-like paratenon is pathological, we are unaware of any evidence supporting this interpretation.

It is well known that knee joints undergo frequent movements in utero $[6,7,11]$. A baby usually has a knee flexion posture at birth. In new-borns and infants, stiffness at the knee is released from 21.4 to 3.3 degrees within the first 6 months [2]. This knee stiffness is apparently caused by a mass-effect of the thick paratenon armoured around the cruciate ligaments. A slight twisting or rotation at the knee is likely to release an infant's knee because it can break the paratenon and accelerate cavitation. Finally, we had a study limitation that we did not perform a MOVAT's pentachrome staining [17] for long-preserved specimens.

\section{CONCLUSIONS}

Differences in knee movements in utero seemed to cause differences in the thickness of the paratenon among foetuses. New-borns might have limited knee flexion due to a mass-effect of the thick paratenon around the cruciate ligaments. A slight twisting or rotation at the knee may help to release the knee, because it can break the foetal paratenon and accelerate cavitation.

\section{Conflict of interest: None declared}

\section{REFERENCES}

1. Benjamin $M$, Kaiser E, Milz S. Structure-function relationships in tendons: a review. J Anat. 2008; 212(3): 211-228, doi: 10.1111/j.1469-7580.2008.00864.x, indexed in Pubmed: 18304204.

2. Broughton NS, Wright J, Menelaus MB. Range of knee motion in normal neonates. J Pediatr Orthop. 1993; 13(2): 263-264, indexed in Pubmed: 8459024.

3. Chahla J, Moatshe G, Cinque ME, et al. Arthroscopic anatomic single-bundle anterior cruciate ligament reconstruction using bone-patellar tendon-bone autograft: pearls for an accurate reconstruction. Arthrosc Tech. 2017; 6(4): e1159-e1167, doi: 10.1016/j.eats.2017.04.001, indexed in Pubmed: 29354412.

4. Clark CR, Ogden JA. Development of the menisci of the human knee joint. Morphological changes and their potential role in childhood meniscal injury. J Bone Joint Surg Am. 1983; 65(4): 538-547, indexed in Pubmed: 6833331.

5. Gray DJ, Gardner E. Prenatal development of the human knee and superior tibiofibular joints. Am J Anat. 1950; 86(2): 235-287, doi: 10.1002/aja.1000860204, indexed in Pubmed: 15410671.

6. Higginbottom J, Bagnall $\mathrm{KM}$, Harris $\mathrm{PF}$, et al. Ultrasound monitoring of fetal movements. A method for assessing fetal development? . Lancet. 1976; 307(7962): 719-721, doi: 10.1016/s0140-6736(76)93092-0.

7. Humphrey T. Some correlations between the appearance of human fetal reflexes and the development of the nervous system. Progr Brain Res. 1964: 93-135, doi: 10.1016/ s0079-6123(08)61273-x.

8. Huisman ES, Andersson G, Scott A, et al. Regional molecular and cellular differences in the female rabbit Achilles tendon complex: potential implications for understanding responses to loading. J Anat. 2014; 224(5): 538-547, doi: 10.1111/joa.12169, indexed in Pubmed: 24571598.

9. Isogai S, Murakami G, Wada T, et al. Which morphologies of synovial folds result from degeneration and/or aging of the radiohumeral joint: an anatomic study with cadavers and embryos. J Shoulder Elbow Surg. 2001; 10(2): 169-181, doi: 10.1067/mse.2001.112956, indexed in Pubmed: 11307082.

10. Katz K, Naor N, Merlob P, et al. Rotational deformities of the tibia and foot in preterm infants. J Pediatr Orthop. 1990; 10(4): 483-485, indexed in Pubmed: 2358486.

11. Katz K, Mashiach R, Bar On A, et al. Normal range of fetal knee movements. J Pediatr Orthop. 1999; 19(6): 739-741, indexed in Pubmed: 10573342.

12. Khan KM, Maffulli N, Coleman BD, et al. Patellar tendinopathy: some aspects of basic science and clinical management. Br J Sports Med. 1998; 32(4): 346-355, doi: 10.1136/bjsm.32.4.346, indexed in Pubmed: 9865413.

13. Kitamura K, Hayashi S, Jin ZWu, et al. Fetal cervical zygapophysial joint with special reference to the associated synovial tissue: a histological study using near-term human fetuses. Anat Cell Biol. 2021; 54(1): 65-73, doi: 10.5115/ acb.20.265, indexed in Pubmed: 33594011.

14. Lalam RK, Winn N, Cassar-Pullicino VN. Interventional articular and para-articular knee procedures. Br J Radiol. 2016; 89(1059): 20150413, doi: 10.1259/bjr.20150413, indexed in Pubmed: 26682669.

15. Mérida-Velasco J, Sánchez-Montesinos I, Espín-Ferra J, et al. Development of the human knee joint ligaments. Anat Rec. 1997; 248(2): 259-268, doi: 10.1002/(sici)10970185(199706)248:2<259::aid-ar13>3.0.co;2-o.

16. McDermott L. Development of the human knee joint. Arch Surg. 1943; 46(5): 705, doi: 10.1001/archsurg.1943.01220110121021.

17. Movat $\mathrm{HZ}$, More RH. The nature and origin of fibrinoid. Am J Clin Pathol. 1957; 28(4): 331-353, doi: 10.1093/ ajcp/28.4.331, indexed in Pubmed: 13478538.

18. Shaw HM, Vázquez OT, McGonagle D, et al. Development of the human Achilles tendon enthesis organ. J Anat. 2008; 213(6): 718-724, doi: 10.1111/j.1469-7580.2008.00997.x, indexed in Pubmed: 19094187.

19. Stecco C, Corradin M, Macchi V, et al. Plantar fascia anatomy and its relationship with Achilles tendon and paratenon. J Anat. 2013; 223(6): 665-676, doi: 10.1111/ joa.12111, indexed in Pubmed: 24028383.

20. Takayama T, Hirano-Kawamoto Ai, Yamamoto $M$, et al. Macrophage infiltration into thyroid follicles: an immunohistochemical study using donated elderly cadavers. Okajimas Folia Anat Jpn. 2016; 93(3): 73-80, doi: 10.2535/ ofaj.93.73, indexed in Pubmed: 28216539.

21. Uchiyama Y, Oomiya A, Murakami G. Fluctuations in follicular structures of rat thyroid glands during 24 hours: fine structural and morphometric studies. Am J Anat. 1986; 175(1): 23-33, doi: 10.1002/aja.1001750104, indexed in Pubmed: 3953468. 